\section{Irreversible electroporation of hepatocellular carcinoma: the role of ultrasonography}

\author{
Katsutoshi Sugimoto, Masakazu Abe, Yu Yoshimasu, Hirohito Takeuchi, Yoshitaka Kasai, \\ Takao Itoi
}

Department of Gastroenterology and Hepatology, Tokyo Medical University, Tokyo, Japan

Irreversible electroporation (IRE) is a novel form of soft tissue ablation therapy that uses highcurrent electrical pulses to induce the formation of pores in the cell membrane, leading to cell death. Although outcome data for the ablation of hepatocellular carcinoma (HCC) by IRE are limited, early results are encouraging and may suggest equivalency to the outcomes achieved by thermal ablation methods such as radiofrequency ablation (RFA) and microwave ablation (MWA). However, IRE can be a challenging and very time-consuming procedure compared to RFA and MWA. In this review article, we not only evaluate the efficacy and safety of IRE for the treatment of HCC, but also discuss imaging guidance, ablation monitoring, and endpoint assessment, with a particular focus on ultrasonography.

Keywords: Irreversible electroporation; Hepatocellular carcinoma; Ultrasonography; Ablation

\section{Introduction}

According to the World Health Organization, hepatocellular carcinoma (HCC) is the fifth most common tumor worldwide and the second most common cause of cancer-related death (http:// globocan.iarc.fr/old/FactSheets/cancers/liver-new.asp). Thermal ablation using either radiofrequency ablation (RFA) or microwave ablation (MWA) has been established as standard-of-care therapy for small HCCs that are not amenable to surgical resection [1]. However, the use of thermal ablation is limited by both the heat sink effect and the risk of collateral damage to heat-sensitive structures [2].

Recently, irreversible electroporation (IRE) has emerged in the field of interventional oncology. This is a nonthermal form of tumor ablation that is unaffected by the heat sink effect and may cause less collateral damage based on its mechanism of action [3]. Specifically, IRE relies on ultrashort highvoltage electrical pulses to induce the formation of pores in the lipid bilayer of cells, leading to cell death via apoptosis. Collagenous structures within the ablation field are spared, thereby preserving parenchymal architecture [4].

The only commercially available IRE system is NanoKnife (AngioDynamics, Queensbury, NY, USA) (Fig. 1). The electrodes are housed in a 19-gauge probe. The system supports the simultaneous use of two to six monopolar electrodes, which can be positioned under either ultrasound or computed tomography (CT) guidance. The distribution of the electric field depends on the positions of the

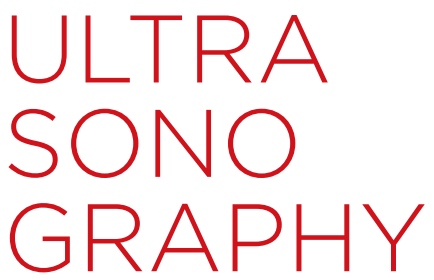

\section{REVIEW ARTICLE}

https://doi.org/10.14366/usg.20023 pISSN: 2288-5919 - elSSN: 2288-5943

Ultrasonography 2020;39:229-237

Received: February 13, 2020

Revised: April 2, 2020

Accepted: April 2, 2020

Correspondence to:

Katsutoshi Sugimoto, MD, Department of Gastroenterology and Hepatology, Tokyo Medical University, 6-7-1

Nishishinjuku, Shinjuku-ku, Tokyo 160-0023, Japan

Tel. +81-(0)3-3342-6111

Fax. +81-(0)3-5381-6654 E-mail: sugimoto@tokyo-med.ac.jp

This is an Open Access article distributed under the terms of the Creative Commons Attribution NonCommercial License (http://creativecommons.org/ licenses/by-nc/4.0/) which permits unrestricted noncommercial use, distribution, and reproduction in any medium, provided the original work is properly cited.

Copyright (C) 2020 Korean Society of Ultrasound in Medicine (KSUM)

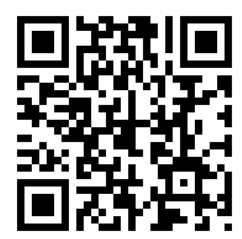

How to cite this article:

Sugimoto K, Abe M, Yoshimasu Y, Takeuchi $\mathrm{H}$, Kasai $Y$, Itoi T. Irreversible electroporation of hepatocellular carcinoma: the role of ultrasonography. Ultrasonography. 2020 Jul;39(3):229-237. 


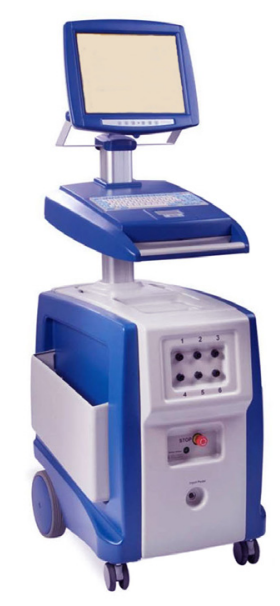

A

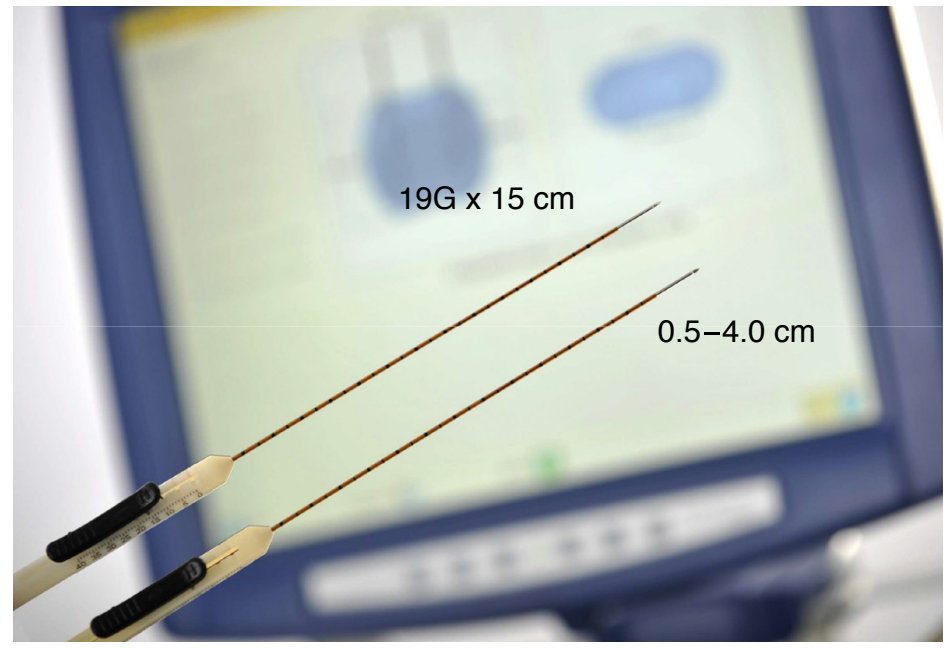

B

Fig. 1. Outer appearance of the NanoKnife irreversible electroporation system.

A. NanoKnife generator. B. NanoKnife electrodes: the active working length of each electrode is adjustable from 0.5 to $4.0 \mathrm{~cm}$. The active working length is usually set at 1.5 or $2.0 \mathrm{~cm}$ by most physicians when treating liver lesions.

electrodes, as all electrodes must be placed as parallel to each other as possible in order to ensure a homogeneous energy field. Thus, compared to RFA and MWA, electrode placement in IRE can be very challenging due to the need to ensure the precise parallel placement of multiple electrodes. Moreover, the relative instability of the 19-gauge electrodes used for IRE compared to the needles used for MWA and RFA makes it more difficult to avoid positional deviation.

As mentioned above, IRE can be a challenging and very timeconsuming procedure, especially for the ablation of deep-seated and hard-to-image lesions. In this review article, we not only evaluate the efficacy and safety of IRE for the treatment of HCC, but also discuss IRE electrode insertion and imaging guidance, as well as IRE ablation monitoring and endpoint assessment. Given the specialized nature of this journal, the present review article focuses on the role of ultrasonography in IRE.

\section{Efficacy and Safety of IRE for HCC}

In order to evaluate the efficacy and safety of IRE for the treatment of HCC, we conducted a comprehensive literature search in PubMed using the keywords "irreversible electroporation" and "hepatocellular carcinoma." A total of 82 articles were identified, including original studies on the efficacy, clinical outcomes, and safety profile of IRE for HCC. Studies that investigated secondary liver cancer as well as HCC were also included, provided that they presented a detailed analysis of HCC. After a thorough screening, six studies were selected for inclusion in this article.

The data were extracted by one of the authors. The following items were analyzed: study design, number of patients, number of lesions, approach (percutaneous or operative), guidance, tumor size, local tumor progression (LTP) rate, median follow-up period, complication rate, and number of deaths. The studies are shown in Table 1.

Sutter et al. [5] conducted a retrospective analysis of a series of 58 patients with 75 HCCS treated by IRE. The median tumor size was $24 \mathrm{~mm}$. The locations and types of the HCCs were described as hilar $(n=47,62.7 \%)$, peripheral $(n=13,37.3 \%)$, infiltrative form $(n=7,9.3 \%)$, and portal invasion $(n=10,13.3 \%)$, with some overlap. All of these HCCs were not amenable to resection or thermal ablative therapies because of a high risk of major complications. The authors employed a percutaneous approach in all patients, with guidance by ultrasonography alone or by ultrasonography fused with cone-beam CT. After a median follow-up period of 9 months (range, 0.3 to 31 months), LTP was detected in $15(20 \%)$ of the 69 tumors that appeared to have been completely ablated (69 of the 75 tumors, 92\%). With regard to safety, according to the ClavienDindo classification, 11 patients (19\%) experienced complications (8 patients experienced minor complications [all grade II or III] and three patients experienced major complications [2 grade III and 1 grade IV]). There were no complications related to bile duct injury.

Kalra et al. [6] conducted a retrospective study including 21 patients with $21 \mathrm{HCCS}$ treated by IRE. The median tumor size was $26 \mathrm{~mm}$. The locations of the HCCs were described as central $(n=13$, $62 \%)$, peripheral $(n=8,38 \%)$, in proximity to large vessels $(n=10$, $48 \%)$, in proximity to hilar bile duct/gallbladder $(n=3,14 \%)$, and in proximity to the diaphragm $(n=7,33 \%)$, with some overlap. The 
Table 1. Outcomes of IRE studies on HCC

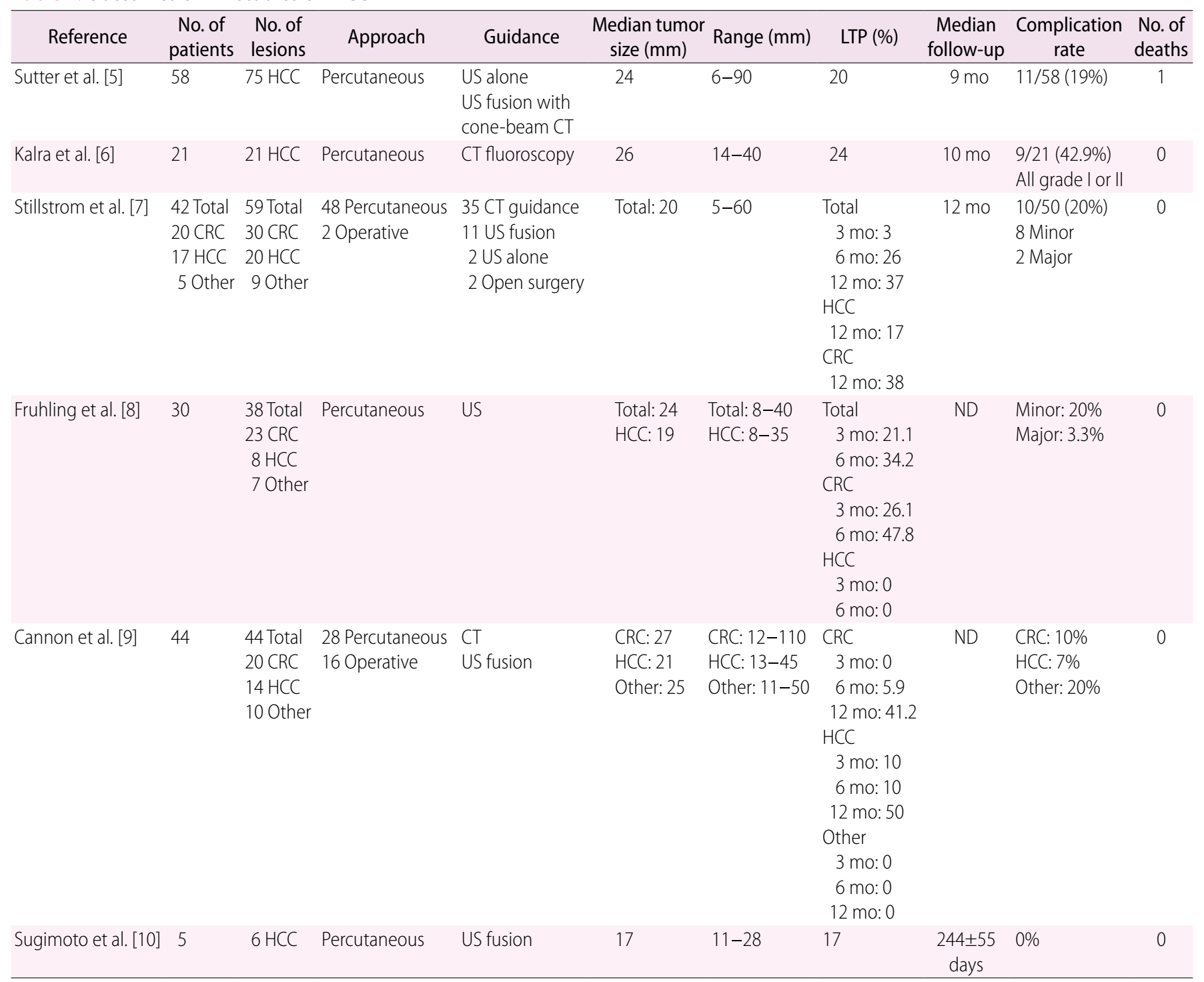

IRE, irreversible electroporation; HCC, hepatocellular carcinoma; LTP, local tumor progression; US, ultrasonography; CT, computed tomography; CRC, colorectal cancer; ND, not detected.

authors employed a percutaneous approach in all patients, with guidance by CT fluoroscopy. After a median follow-up period of 10 months (range, 2 to 30 months), LTP was detected in five of the 21 tumors (24\%). Complications were observed in nine patients (42.9\%) and were classified as grade 1 or 2 (i.e., minor) based on the CIRSE recommendations for the evaluation of complications. There were no complications related to bile duct injury or procedurerelated deaths.

Stillstrom et al. [7] conducted a retrospective study involving 42 patients with 59 malignant liver tumors treated by IRE. Their study group included 17 patients with 20 HCCs. The median tumor size was $20 \mathrm{~mm}$ (including both HCCs and other types of liver tumors). Although the study included tumors that were located too close to central bile ducts and/or portal branches to allow safe thermal ablation, the specific locations were not described. The authors employed a percutaneous approach for 48 procedures and an operative approach for two procedures, with guidance by $\mathrm{CT}$ using the GAS-ONE stereotactic CT-guided navigation system ( $n=35)$, by ultrasound fusion $(n=11)$, by ultrasonography alone $(n=2)$, or by open surgery $(n=2)$. The LTP rate for HCCs at 12 months was $17 \%$. Procedural complications occurring within 30 days were observed in 10 patients (20\%). Eight of the complications were ClavienDindo grade I-IIIa and two were grade IIIb-IVb. There were no complications related to bile duct injury. There were no deaths up to 30 days post-procedure.

Fruhling et al. [8] conducted a retrospective study of 30 patients 
with 38 malignant liver tumors treated by IRE. Their study group included eight patients with eight HCCs. The median size of the HCCs was $19 \mathrm{~mm}$. Although the study included tumors that were located close to large vessels and/or biliary ducts, meaning that surgical resection or treatment with other forms of ablation was contraindicated, the specific locations were not described. The authors employed a percutaneous approach in all patients, with guidance by ultrasonography. Among the patients with HCC, there were no local recurrences at 3 and 6 months and no complications or procedure-related deaths.

Cannon et al. [9] conducted a prospective study involving 44 patients with 44 malignant liver tumors treated by IRE. Their study group included 14 patients with 14 HCCs. The median tumor size of the HCCs was $21 \mathrm{~mm}$. The locations of the HCCs were not described. The authors employed either a percutaneous or operative approach. The percutaneous procedures were performed under CT guidance. The LTP rates for HCCs were $10 \%$ at 3 months, $10 \%$ at 6 months, and $50 \%$ at 12 months. One minor complication was observed in one patient with HCC, and there were no deaths up to 30 days post-procedure. There were no complications related to bile duct injury.

Sugimoto et al. [10] conducted a prospective study of five patients with six HCCs treated by IRE. The median tumor size was $17 \mathrm{~mm}$. The tumors were not all located close to large vessels and/or biliary ducts, so surgical resection or treatment with other forms of ablation was not necessarily contraindicated, but IRE was performed because the main purpose of the study was to assess the safety of the technique. The authors employed a percutaneous approach under ultrasound guidance in all patients. The LTP rate was $17 \%$, and there were no complications or procedure-related deaths.

To summarize the HCC-specific findings of the above studies, all six of the studies most commonly employed a percutaneous approach. Three studies mainly used ultrasound guidance, and the other three studies mainly used CT guidance. The median (or mean) tumor sizes ranged from 17.5 to $26 \mathrm{~mm}$. The LTP rates ranged from $0 \%$ to $24 \%$ at 6 months. The complication rates ranged from $0 \%$ to $42.9 \%$, with almost all complications classified as minor. These reported outcomes were safely achieved in the treatment of lesions located in close proximity to either portal structures or large vessels, which is the most notable advantage of IRE. Although the LTP rates were higher than those reported for thermal ablation methods such as RFA and MWA [1], this comparison is not fundamentally valid because IRE is usually selected for patients with tumors in challenging locations that are difficult to treat using thermal ablation methods. Moreover, IRE procedures are more complicated than RFA and MWA procedures because IRE requires the insertion of multiple electrodes, which must be positioned as parallel to each other as possible. Thus, to achieve the best possible therapeutic effectiveness in IRE, we must have a clear understanding of its imaging features.

\section{Comparison with Other Modalities}

Due to the nature of IRE, prospective head-to-head comparisons with other modalities such as RFA and MWA are difficult to perform. To our knowledge, only two papers have described the efficacy and/or safety of IRE compared to RFA and MWA in a retrospective setting. One paper is that of Bhutiani et al. [11], who compared the efficacy of IRE and MWA for the treatment of HCC. A total of 55 patients underwent either IRE $(n=30)$ or MWA $(n=25)$ for Child-Pugh B (7/8) HCC. The decision to perform IRE or MWA was based on the anatomical location of the tumor and its proximity to major vascular and biliary structures. Both modalities were found to have similar treatment success rates: $100 \%$ and $100 \%$ at 90 days and $97 \%$ and $100 \%$ at 180 days $(P=0.37)$ for IRE and MWA, respectively. The advantage of IRE over MWA was that patients who underwent IRE had a shorter length of stay $(\mathrm{P}=0.05)$ and a lower 90-day readmission rate $(P=0.03)$ than those who underwent MWA.

Verloh et al. [12] compared the frequency of adverse events related to IRE and RFA/MWA in patients with HCC. They found that $34 \%$ (16 of 47) of patients who underwent IRE and $26.5 \%$ (31 of 117) of patients who underwent RFA/MWA experienced grade I or II adverse events. The major complication rates were also comparable between the two groups: $2.1 \%$ ( 1 of 47 ) and 2.6\% (3 of 117) in the IRE and RFA/MWA groups, respectively. These results suggest that IRE and RFA/MWA have comparable complication rates despite the larger number of punctures in IRE procedures.

The following section discusses ultrasound guidance, intraprocedural monitoring, and post-IRE ultrasound imaging findings.

\section{Electrode Insertion and Ultrasound Guidance}

The main challenge in IRE electrode placement is that multiple electrodes must be placed parallel to each other while separated by a distance of 1.5 to $2.0 \mathrm{~cm}$. The relative instability of the 19-gauge electrodes used in IRE compared to the needles used in MWA or RFA makes it more difficult to avoid positional deviation. Thus, the successful ablation of deep-seated and hard-to-image lesions is highly dependent on the experience of the interventionalist performing the procedure.

When ultrasound guidance is used for electrode placement, electromagnetic tracking-based fusion imaging with CT or magnetic resonance (MR) images (Smart-Fusion, Canon Medical Systems, 
Tochigi, Japan) can be a valuable tool [13]. The fusion images show the same plane as the ultrasound image and are displayed in real time. For lesions that are difficult to visualize using conventional B-mode imaging or even contrast-enhanced ultrasound (CEUS), the generation of fusion images can improve lesion detectability [14] (Fig. 2).

To overcome the challenges associated with the placement of multiple electrodes using ultrasonography, it may be helpful to employ a 3-dimensional (3D) simulator system (3D Sim-Navigator, Hitachi, Tokyo, Japan), which can superimpose multiple virtual puncture lines on 3D-CT or magnetic resonance imaging (MRI). Moreover, to facilitate the accurate insertion of multiple electrodes in a parallel manner to the appropriate depth, the system can display the "C-plane" (the plane perpendicular to the electrode line) in addition to the "B-plane" (the normal ultrasound plane). The simulator system also shows the distances between the electrodes, which is essential for successful IRE treatment [15] (Fig. 3).

The use of a needle-tracking system (Smart Navigation, Canon Medical Systems, Tochigi, Japan) is helpful for highlighting the locations of the electrode tips. This is because IRE electrodes are thinner than RFA and MWA needles, making it more difficult to visualize the tips [16] when they are placed deep in a coarse cirrhotic liver. The needle-tracking system makes it much easier to treat lesions located in close proximity to either portal structures or large vessels because it can clearly display the electrode tips and show the relationships between the electrode tips and these vital structures. When pull-back ablation is performed, the system is also helpful for ensuring that the electrodes are pulled back by the correct distance (Fig. 4).

Despite the advantages discussed above, ultrasound-guided IRE suffers from a number of limitations. One major limitation is that when a lesion cannot be visualized because it is in a difficult location, CT guidance is preferable to ultrasound guidance. However, this situation is relatively rare because artificial ascites and pleural effusion make it possible to detect such lesions in many patients [17]. Another limitation of ultrasonography is that it is not suitable for whole-body scanning. The use of $\mathrm{CT}$ is mandatory for the detection of complications such as pneumothorax or perforation of the Gl tract.

\section{Ablation Monitoring and Endpoint Assessment Using Ultrasonography}

Intraprocedural monitoring and post-ablation imaging are key factors in successful ablation. It is of the utmost importance to review post-IRE imaging findings to assess the technical efficacy of the technique, as well as to ensure the long-term success of ablation and to allow the early detection of LTP post-ablation. Unfortunately,

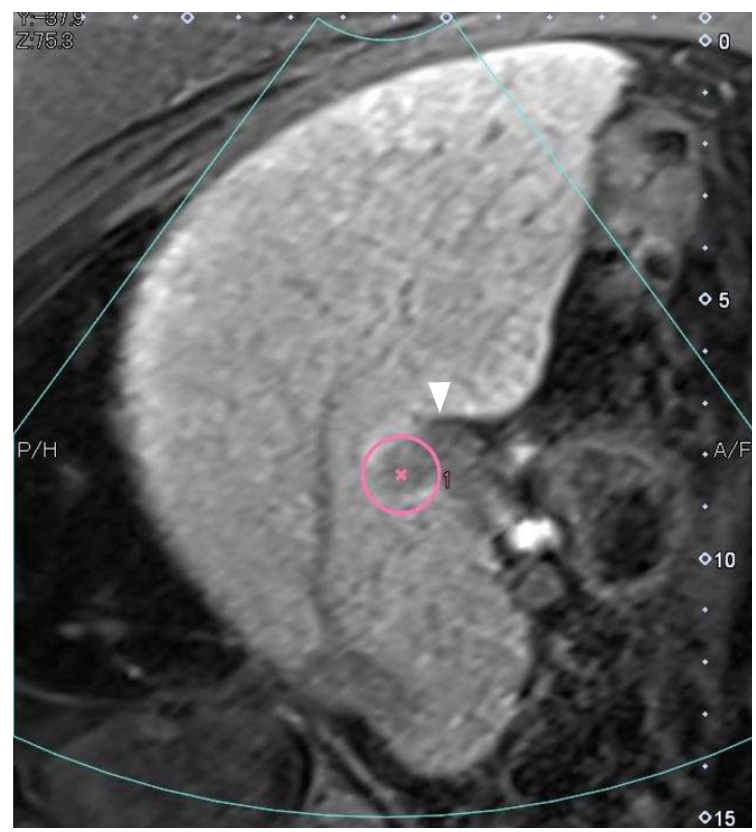

$A$

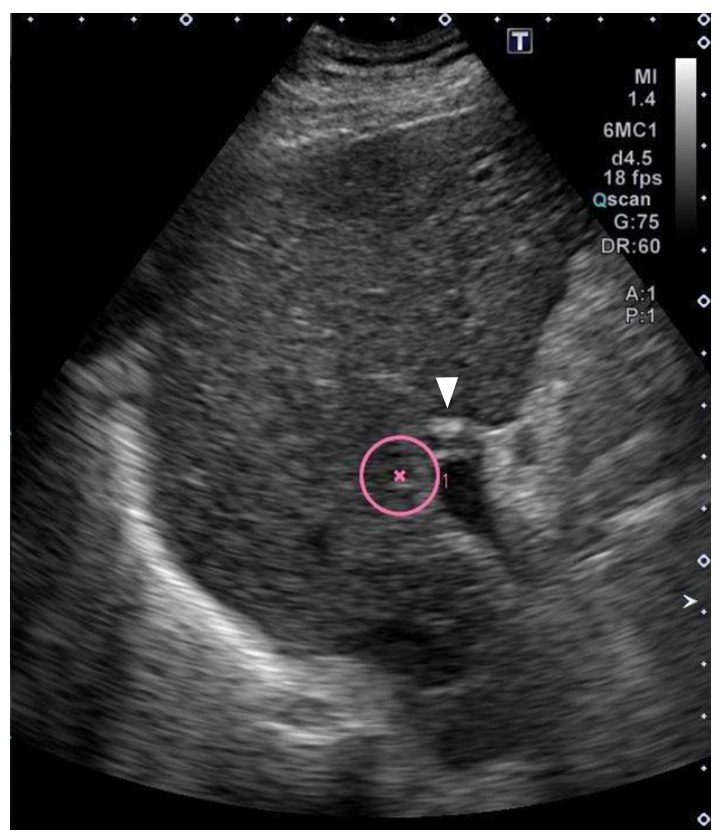

B

Fig. 2. EOB-MRI-US fusion image from a 76-year-old man with HCC (pink circle) adjacent to the anterior segment branch of the portal vein (arrowheads).

A. The left-side image demonstrates HCC as a clear hypointense nodule on the hepatobiliary phase of EOB-MRI. B. The rightside image demonstrates HCC as an obscure slightly hypoechoic nodule on B-mode US. EOB-MRI, gadolinium ethoxybenzyldiethylenetriaminepentaacetic acid-enhanced magnetic resonance imaging; US, ultrasonography; HCC, hepatocellular carcinoma. 


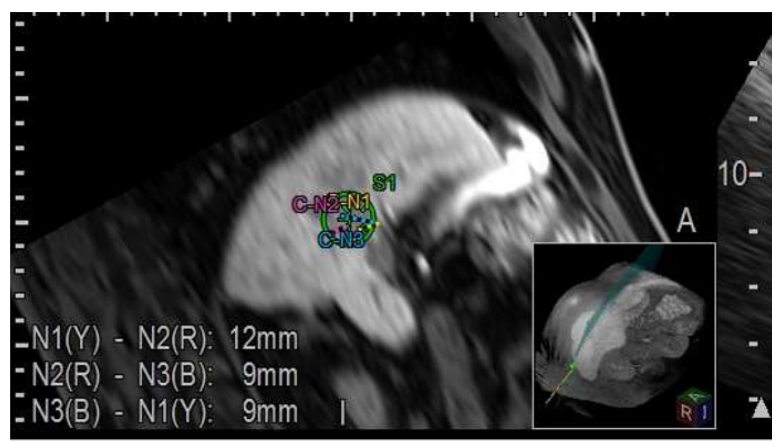

A
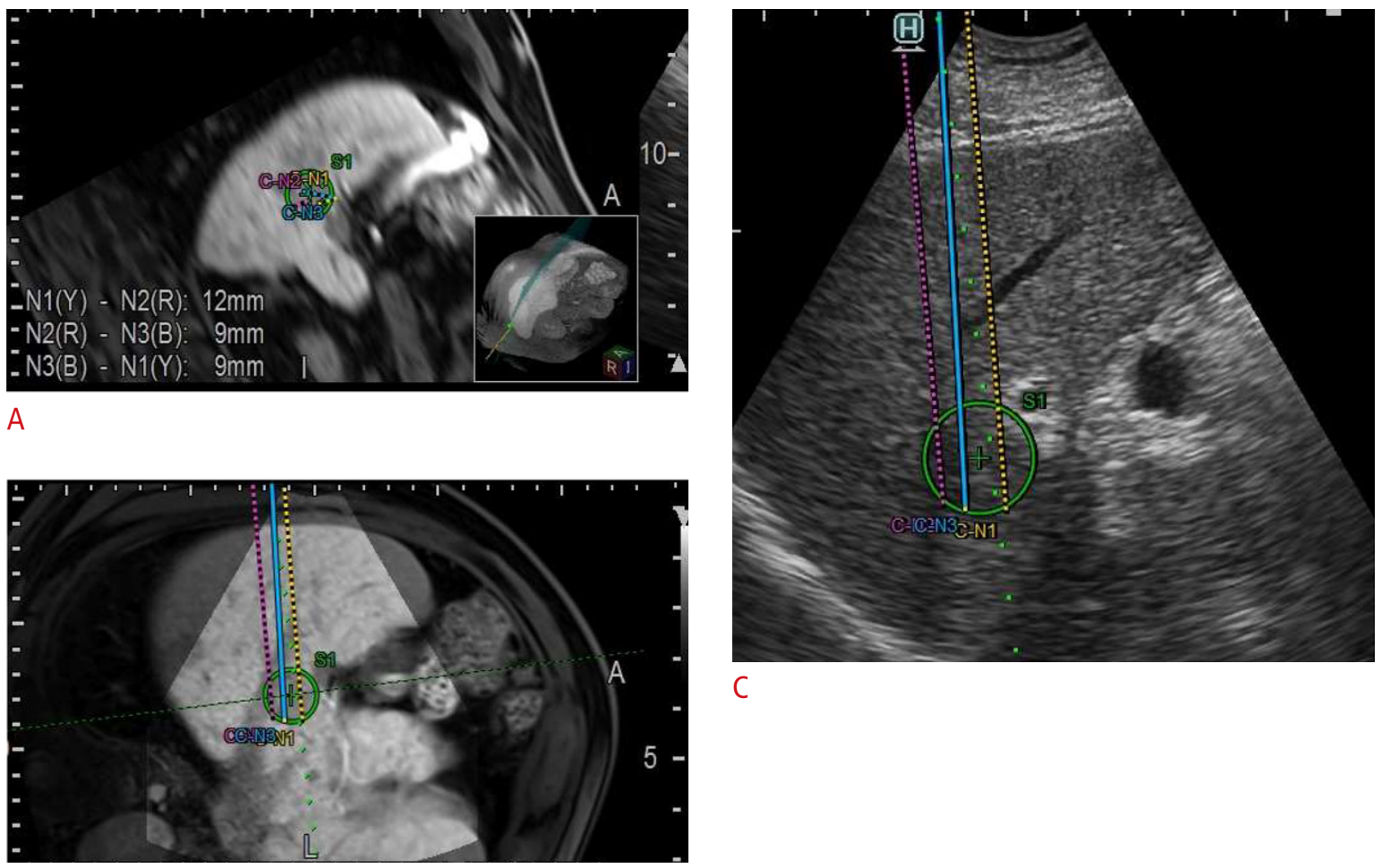

C

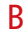

Fig. 3. EOB-MRI-US fusion image from a 73-year-old man with hepatocellular carcinoma (green circle).

The image shows a simulation of the insertion of three IRE electrodes. A. The system can display the "C-plane," which corresponds to the plane perpendicular to the electrode line. The distances between the electrodes are shown in the lower left corner. B. The system can also display the "B-plane," which corresponds to the standard US plane (C). EOB-MRI, gadolinium ethoxybenzyl-diethylenetriaminepentaacetic acid-enhanced magnetic resonance imaging; US, ultrasonography; IRE, irreversible electroporation.

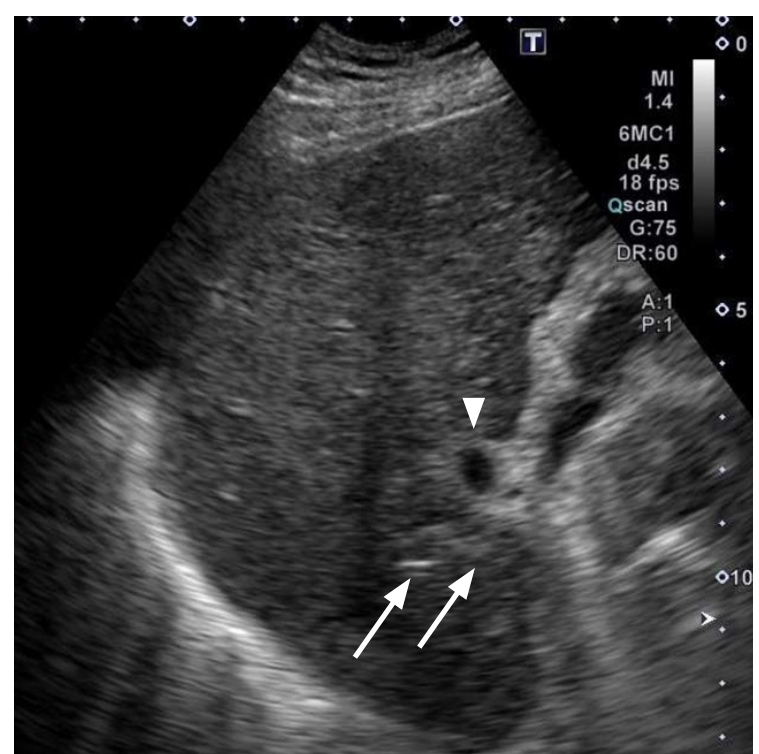

A

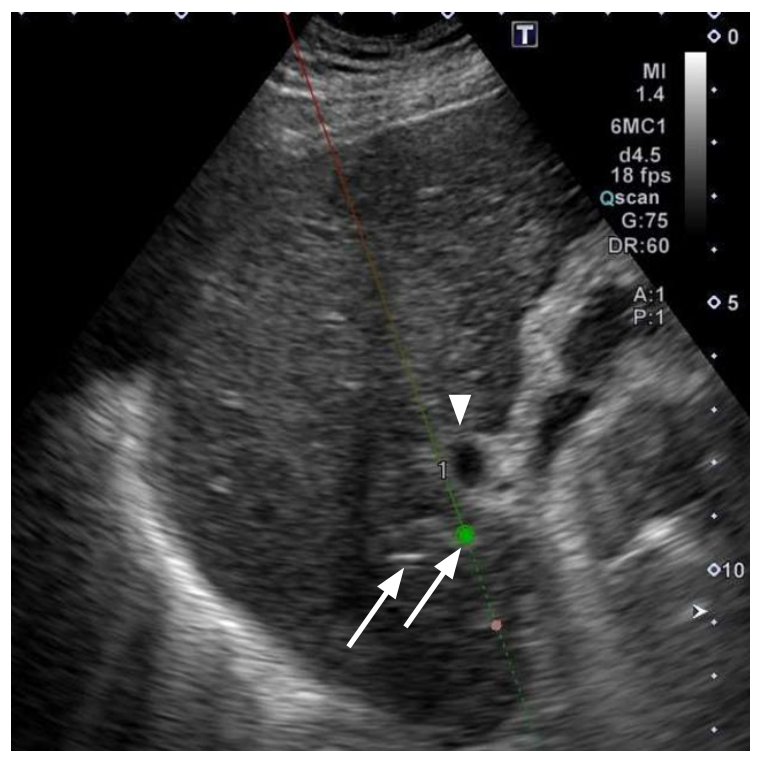

B

Fig. 4. Ultrasonography from a 76-year-old man with HCC during IRE (same case as Fig. 2).

A. B-mode ultrasonography shows two IRE electrodes inserted into the HCC adjacent to an anterior segment branch of the portal vein (arrowhead). Although the electrode tips are slightly hyperechoic (arrows), they are difficult to see. B. Using the needle-tracking system, both the needle line (arrows) and the needle tip (green ball) are clearly highlighted. HCC, hepatocellular carcinoma; IRE, irreversible electroporation. 
the specific imaging findings after IRE are still poorly understood, which is one possible reason for its lower success rate in achieving complete local tumor eradication. Specifically, unlike thermal ablation methods such as RFA and MWA, persistent enhancement of the peritumoral liver parenchyma is observed within the IRE ablation zone by contrast-enhanced imaging (CT, MRI, and ultrasonography), and the ablation margins are therefore not clearly defined in IRE.

The practicability of real-time ultrasound monitoring has been confirmed in animal and human studies. Typically, a small area of gas forms around each electrode tip, probably caused by the electrolysis of water into hydrogen and oxygen gas. This appears as a small hyperechoic area in B-mode ultrasound images. Depending on the electroporation protocol employed, these small gas bubbles may also expand to fill part or all of the ablation zone.

Appelbaum et al. [18] reported temporal changes within the IRE ablation zone in B-mode ultrasound images of normal pig liver. Immediately after ablation, the ablation zone appeared as a hypoechoic area. Over the next 15 minutes, the zone became progressively more isoechoic, and a peripheral hyperechoic rim started to form 90-120 minutes after ablation. This peripheral rim was shown to best correlate with the pathologic findings of necrosis and/or apoptosis in the ablation zone. Although these B-mode findings are interesting, they are not directly applicable in actual clinical practice, particularly for the assessment of IRE treatment efficacy for HCC.

Niessen et al. [19] reported temporal changes in CEUS findings for HCC before and after IRE. After IRE, all HCCs showed complete devascularization, with a hyper-enhancing rim observed around the ablation defects during the arterial phase on CEUS. The ablation defects showed significant shrinkage and reduced peripheral enhancement over the course of follow-up. The authors speculated that the hyper-enhancing rim observed around the ablation defects might represent a perifocal zone of reversible electroporation.

Sugimoto et al. [20] reported the relationship between post-IRE CEUS findings and LTP and also compared the findings obtained by 3 imaging modalities: CEUS, contrast-enhanced CT, and gadolinium ethoxybenzyl-diethylenetriaminepentaacetic acid-enhanced magnetic resonance imaging (EOB-MRI). They concluded that the best way to determine whether or not lesions have been completely treated is to observe a loss of intratumoral enhancement in hypervascular tumors. For this purpose, CEUS was judged to be superior to contrast-enhanced CT and EOB-MRI due to its higher sensitivity for blood flow and because microbubbles are pure intravascular tracers. Thus, the treatment endpoint of IRE should be to observe a loss of intratumoral enhancement, regardless of the presence of a safety margin (Fig. 5). To interventionalists, this is a crucial finding

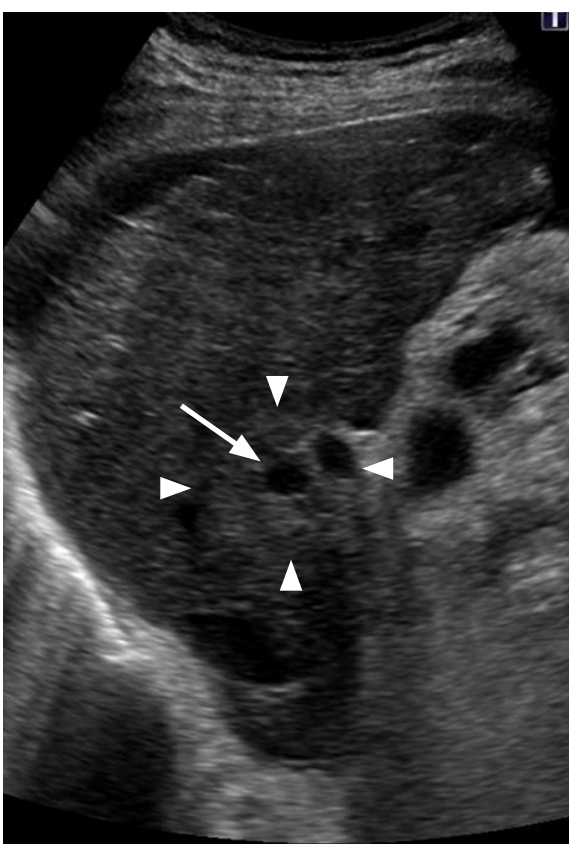

A

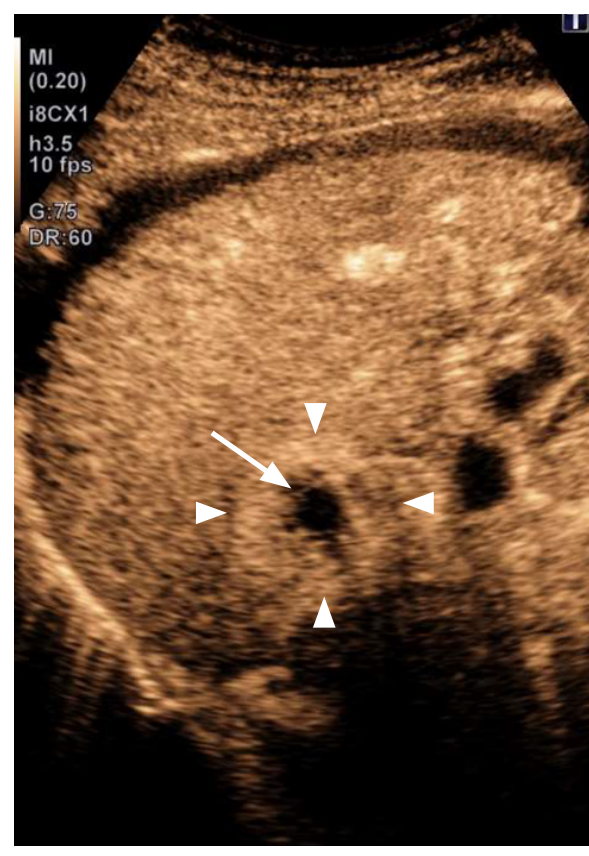

B

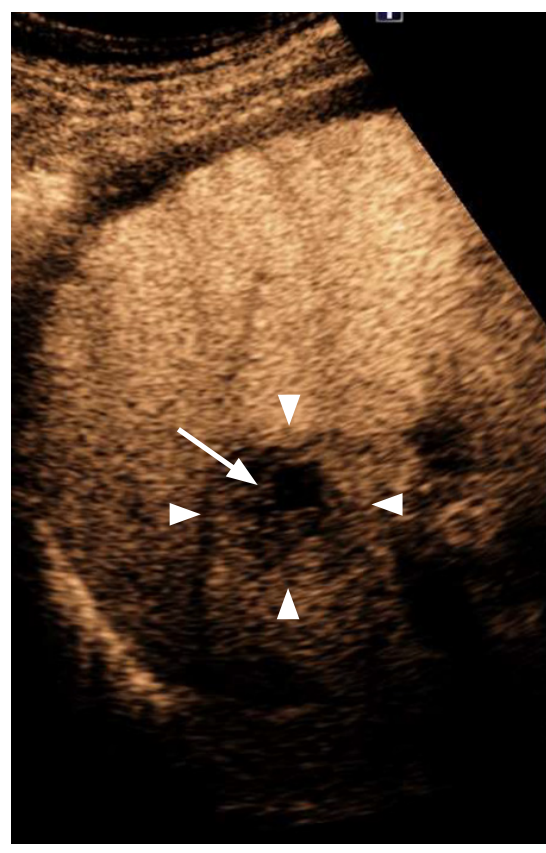

C

Fig. 5. Images from a 76-year-old man at 1 day after IRE for HCC (same case as Fig. 2).

A. A B-mode ultrasonography shows that the HCC was hypoechoic (arrow), surrounded by a hyperechoic area (arrowheads). B. The arterial phase of contrast-enhanced ultrasonography (using Sonazoid) showed that the HCC was avascular (arrow) with rim enhancement (arrowheads). Portal vein flow was not affected. C. A Kupffer phase image showed that the HCC was in an area of contrast defect (arrow), and the rim-enhanced area was slightly hypoechoic (arrowheads). IRE, irreversible electroporation; HCC, hepatocellular carcinoma. 
for identifying residual tumor tissue immediately after IRE and for planning re-treatment. CEUS is therefore considered the most effective modality for intraprocedural monitoring and evaluating the technical success of IRE.

\section{Conclusion}

IRE is a relatively new nonthermal ablation technique. It has been found to be useful for the treatment of HCCS that are not amenable to either surgical resection or thermal ablation methods such as RFA and MWA. Based on the findings of a literature review, IRE is considered to be more useful for small HCCs, and the complications associated with this technique appear to be comparable to those of other ablation methods. Ultrasonography plays an important role in IRE, not only for imaging guidance, but also for ablation monitoring and endpoint assessment to achieve complete local tumor eradication. Further research is needed to gain clearer insights into the efficacy and safety of this therapeutic technique.

ORCID: Katsutoshi Sugimoto: https://orcid.org/0000-0001-6271-7806; Masakazu Abe: https://orcid.org/0000-0002-8442-3216; Yu Yoshimasu: https://orcid.org/00000003-0818-2294; Hirohito Takeuchi: https://orcid.org/0000-0002-2082-3202; Yoshitaka Kasai: https://orcid.org/0000-0003-4396-6166; Takao Itoi: https://orcid.org/0000-00029433-8437

\section{Author Contributions}

Conceptualization: Sugimoto K, Itoi T. Drafting of the manuscript: Sugimoto K. Critical revision of the manuscript: Abe M, Yoshimasu Y, Takeuchi $\mathrm{H}$, Kasai Y. Approval of the final version of the manuscript: all authors.

\section{Conflict of Interest}

No potential conflict of interest relevant to this article was reported.

\section{References}

1. Facciorusso A, Di Maso M, Muscatiello N. Microwave ablation versus radiofrequency ablation for the treatment of hepatocellular carcinoma: a systematic review and meta-analysis. Int J Hyperthermia 2016;32:339-344.

2. Kondo Y, Shiina S, Tateishi R, Arano T, Uchino K, Enooku K, et al. Intrahepatic bile duct dilatation after percutaneous radiofrequency ablation for hepatocellular carcinoma: impact on patient's prognosis. Liver Int 2011;31:197-205.

3. Rubinsky B, Onik G, Mikus P. Irreversible electroporation: a new ablation modality: clinical implications. Technol Cancer Res Treat 2007;6:37-48.
4. Lee EW, Chen C, Prieto VE, Dry SM, Loh CT, Kee ST. Advanced hepatic ablation technique for creating complete cell death: irreversible electroporation. Radiology 2010;255:426-433.

5. Sutter O, Calvo J, N'Kontchou G, Nault JC, Ourabia R, Nahon P, et al. Safety and efficacy of irreversible electroporation for the treatment of hepatocellular carcinoma not amenable to thermal ablation techniques: a retrospective single-center case series. Radiology 2017;284:877-886.

6. Kalra N, Gupta P, Gorsi U, Bhujade H, Chaluvashetty SB, Duseja A, et al. Irreversible electroporation for unresectable hepatocellular carcinoma: initial experience. Cardiovasc Intervent Radiol 2019;42:584-590.

7. Stillstrom D, Beermann M, Engstrand J, Freedman J, Nilsson H. Initial experience with irreversible electroporation of liver tumours. Eur J Radiol Open 2019;6:62-67.

8. Fruhling P, Nilsson A, Duraj F, Haglund U, Noren A. Single-center nonrandomized clinical trial to assess the safety and efficacy of irreversible electroporation (IRE) ablation of liver tumors in humans: short to mid-term results. Eur J Surg Oncol 2017;43:751-757.

9. Cannon R, Ellis $S$, Hayes D, Narayanan G, Martin RC 2nd. Safety and early efficacy of irreversible electroporation for hepatic tumors in proximity to vital structures. J Surg Oncol 2013;107:544-549.

10. Sugimoto K, Moriyasu F, Kobayashi Y, Saito K, Takeuchi H, Ogawa S, et al. Irreversible electroporation for nonthermal tumor ablation in patients with hepatocellular carcinoma: initial clinical experience in Japan. Jpn J Radiol 2015;33:424-432.

11. Bhutiani N, Philips P, Scoggins CR, McMasters KM, Potts $M H$, Martin RC. Evaluation of tolerability and efficacy of irreversible electroporation (IRE) in treatment of Child-Pugh B (7/8) hepatocellular carcinoma (HCC). HPB (Oxford) 2016;18:593-599.

12. Verloh $N$, Jensch I, Lurken $L$, Haimerl $M$, Dollinger $M$, Renner $P$, et al. Similar complication rates for irreversible electroporation and thermal ablation in patients with hepatocellular tumors. Radiol Oncol 2019;53:116-122.

13. Lee MW, Rhim H, Cha DI, Kim YJ, Lim HK. Planning US for percutaneous radiofrequency ablation of small hepatocellular carcinomas $(1-3 \mathrm{~cm})$ : value of fusion imaging with conventional US and CT/MR images. J Vasc Interv Radiol 2013;24:958-965.

14. Minami Y, Kudo M, Hatanaka K, Kitai S, Inoue T, Hagiwara S, et al. Radiofrequency ablation guided by contrast harmonic sonography using perfluorocarbon microbubbles (Sonazoid) for hepatic malignancies: an initial experience. Liver Int 2010;30:759-764.

15. Hirooka M, Koizumi Y, Imai Y, Nakamura Y, Yukimoto A, Watanabe T, et al. Clinical utility of multipolar ablation with a 3-D simulator system for patients with liver cancer. J Gastroenterol Hepatol 2017;32:1852-1858.

16. Hirooka M, Koizumi Y, Imai Y, Ochi H, Nakamura Y, Yoshida O, et al. Assessment of a needle-tracking system for bipolar radiofrequency ablation. J Med Ultrason (2001) 2016;43:185-191. 
17. Hsieh YC, Limquiaco JL, Lin CC, Chen WT, Lin SM. Radiofrequency ablation following artificial ascites and pleural effusion creation may improve outcomes for hepatocellular carcinoma in high-risk locations. Abdom Radiol (NY) 2019;44:1141-1151.

18. Appelbaum L, Ben-David E, Sosna J, Nissenbaum Y, Goldberg SN. US findings after irreversible electroporation ablation: radiologicpathologic correlation. Radiology 2012;262:117-125.

19. Niessen C, Beyer LP, Haimerl M, Schicho A, Stroszczynski C,
Wiggermann $\mathrm{P}$, et al. Percutaneous irreversible electroporation of hepatocellular carcinoma: contrast-enhanced ultrasound-findings during 1-year follow-up. Clin Hemorheol Microcirc 2019;72:85-93.

20. Sugimoto K, Moriyasu F, Saito K, Kobayashi Y, Itoi T. Multimodality imaging to assess immediate response following irreversible electroporation in patients with malignant hepatic tumors. J Med Ultrason (2001) 2017;44:247-254. 